\title{
Formación de personal investigador en educación a distancia en México desde una perspectiva interdisciplinaria e histórica
}

\author{
Preparation of Research Staff in Distance Education in Mexico from an \\ Interdisciplinary and Historical Perspective
}

\begin{abstract}
Formação de pessoas investigadoras na educação a distância em México a partir da perspectiva interdisciplinária e histórica
\end{abstract}

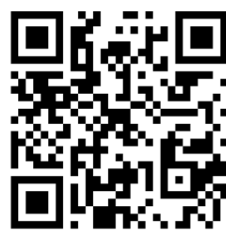

José Antonio Ramírez-Díaz

Universidad de Guadalajara

Guadalajara, México

sigeanton@hotmail.com

https://orcid.org/0000-0003-3354-1842

Recibido $\cdot$ Received $\cdot$ Recebido: 09 / 10 / 2019
Corregido $\cdot$ Revised $\cdot$ Revisado: 08 / 06 / 2021
Aceptado $\cdot$ Accepted $\cdot$ Aprovado: 09 / 07 / 2021

Resumen: El presente artículo se redactó con resultados obtenidos de la investigación: EI CONACYT como organismo rector de las capacidades de Estado en la relación de la ciencia con el sector educativo (Ramírez Díaz, 2020a) que analiza el impacto de la instrumentación de la política científica en la conformación del campo disciplinar educativo en México. El tema revisado en este trabajo relaciona el desarrollo de las disciplinas vinculadas a la educación con los eventos de institucionalización de la educación a distancia. Su objetivo es profundizar en la complejidad del proceso formativo de especialistas de educación a distancia para optimizarlo. El método de investigación se basó en la historiografía y entrevistas a estudiantado egresado de posgrados en educación, los resultados permitieron esbozar límites teóricos a la formación de comunidades académicas especializadas, en las cuales existe un puente entre las demandas sociales y económicas a nivel macro con los procesos regulatorios y la cultura académica de los grupos de formación. Para establecer la relación se hizo uso de la teoría sobre los "social framework". Se reconoció, como limitante, la imposibilidad de situar resultados en forma empírica y dentro de un contexto específico que permitiera caracterizar la manera en la cual operan los "social framework" en los grupos académicos. El trabajo arrojó información teórica para realizar investigación empírica sobre la formación especializada y estableció los campos genéricos de la constitución de comunidades de investigación especializada.

Palabras claves: Educación a distancia; formación; investigación interdisciplinaria; teoría de la educación. 
http://doi.org/10.15359/ree.25-3.13

http://www.una.ac.cr/educare

educare@una.ac.cr

\begin{abstract}
This article was written with results obtained from the research titled CONACYT as a governing body of the state capacities in the relationship of science with the education sector (Ramírez Díaz, 2020a). The article analyzes the impact of the implementation of science policy on the formation of the disciplinary education field in Mexico. The theme reviewed in this paper relates the development of disciplines associated with education to institutionalization events of distance education; its objective is to deepen the complexity in the training process of distance education specialists to optimize it. The research method was based on historiography and interviews with postgraduates in education. The results allowed outlining theoretical limits to the formation of specialized academic communities, in which there is a bridge between social and economic demands at the macro level with regulatory processes and the academic culture of the formation groups. The theory of "Social Framework" was used to establish the relationship. The impossibility of placing results empirically and within a specific context that allows us to characterize how "Social Frameworks" operate in academic groups is recognized as limiting. The work produced theoretical information to conduct empirical research on specialized training and established generic fields of the constitution of specialized research communities.
\end{abstract}

Keywords: Distance education; educational theory; interdisciplinary research; training.

Resumo: Este artigo foi escrito com resultados obtidos com a pesquisa: CONACYT como órgão governamental das capacidades do Estado na relação da ciência com o setor educacional (Ramírez Díaz, 2020a) que analisa o impacto da implementação da política científica na formação do campo: educação disciplinar no México. O tema revisto neste trabalho relaciona o desenvolvimento de disciplinas que involucra à educação com os eventos de institucionalização da educação a distância. Seu objetivo é aprofundar a complexidade do processo de formação de especialistas em educação a distância para otimizá-lo. O método de pesquisa foi historiográfico e entrevistas com pós-graduandos em educação. Os resultados permitiram traçar limites teóricos para a formação de comunidades acadêmicas especializadas, nas quais existe uma ponte entre as demandas sociais e econômicas no nível macro com os processos regulatórios e a cultura acadêmica dos grupos de treinamento Para estabelecer o relacionamento, foi utilizada a teoria do "Marco Social". A impossibilidade de colocar os resultados empiricamente e dentro de um contexto específico que permitisse caracterizar o modo como o Quadro Social opera em grupos acadêmicos foi reconhecida como uma limitante. $O$ trabalho produziu informações teóricas para realizar pesquisas empíricas sobre treinamento especializado e estabeleceu os campos genéricos da constituição de comunidades especializadas em pesquisa.

Palavras-chave: Educação a distância; treinamento; pesquisa interdisciplinar; teoria da educação.

\title{
Introducción
}

La especialización de las disciplinas pauta su desarrollo mediante los procesos de formación para la producción del conocimiento, así como la investigación, aplicación y divulgación de los productos de personal científico consolidado. Estas dinámicas se llevan a cabo en contextos sociales a los cuales se les atribuye la fuerza de cohesión a sus integrantes. Sin embargo, poco se conoce sobre el papel jugado por el desarrollo de las disciplinas y los acontecimientos históricos de los sistemas educativos en los procesos de formación. Este artículo, analiza la importancia de

José Antonio Ramírez-Díaz

Los artículos de la Revista Electrónica Educare del Centro de Investigación y Docencia en Educación de la Universidad Nacional, Costa Rica, se comparten bajo términos de la Licencia Creative Commons: Reconocimiento, № Comercial, Sin Obra Derivada 3.0 Costa Rica. Las autorizaciones adicionales a las aquí delimitadas se pueden obtener en el correo: educare@una.cr 
http://doi.org/10.15359/ree.25-3.13

la conformación entre disciplinas y los acontecimientos históricos como factores vinculantes de la distinción y la formación en el campo disciplinar.

El artículo toma como base la relación entre la sociología, la psicología, la pedagogía y la didáctica con la historia de la institucionalización de la educación en México para, teóricamente, demostrarloselementosinherentes dela construcción deobjetos de investigación enlaeducación a distancia y conformar una propuesta de trabajo que contribuya a identificar elementos finos en la formación. El texto problematiza la incapacidad mostrada por el estudiantado de doctorados en educación de México para precisar los objetos de investigación, ubicarlos en el plano de la interdisciplinariedad y establecer una relación entre la teoría y el recorte de la realidad en sus proyectos. De este problema, se han considerado como causas: la insuficiencia didáctica en la formación de personal investigador (Sánchez Puentes, 1995), el déficit en el capital cultural en el estudiantado (Moreno Bayardo, 2010) y el bajo volumen de investigación en la educación a distancia que impide establecer rutas seguras de formación (Cabero Almenara, 2008; Martín Rodríguez, 1993). También se menciona la escasa calidad de la investigación evidenciada por huecos de información en la bibliografía publicada (Siemens et al., 2015). Ninguno de los estudios referidos incluyó como causas del problema la conformación interdisciplinar de las teorías ni los acontecimientos históricos que dieron impulso a institucionalizar la educación. Esta circunstancia justifica, como planteamiento central del artículo, profundizar en la caracterización y análisis de la complejidad inherente a la relación entre los acontecimientos históricos, el desarrollo teórico, la estructuración de problemas estatales y su incidencia en la formación especializada de personal investigador en la educación a distancia, con el propósito de contribuir a fortalecer la educación superior de esta modalidad. En este artículo se hace un planteamiento en torno a ello.

\section{Marco teórico. El campo de la producción de conocimiento científico}

En el proceso de producción científica, Zemelman $(2004,2011)$ establece una diferencia entre la realidad (histórica) y la teoría. Ilustra una alternativa de construcción del conocimiento por medio del pensar epistémico asumido como un trabajo de resignificación de las teorías en un contexto diferente del cual se generaron. El vínculo entre teoría y realidad remite a un ajuste reflexivo para abordar el objeto de estudio mediante el uso de conceptos resignificados y categorías vinculadas con la realidad específica, considerada por quien investiga. En esta postura, el reto es reconocer un proceso particular de producción de conocimiento que lleva a formar una gran diversidad de comunidades autárquicas e independientes con particulares creencias analíticas, teóricas y epistémicas (Castellanos, 2016).

En la tarea de formación de los personal investigador en educación, el uso de la teoría y el recorte sobre la realidad para explicar los proyectos adoptados (o potenciales) de los actores que son ubicados en la investigación, resulta una tarea con muchas zonas grises. Por su carácter 
http://doi.org/10.15359/ree.25-3.13

http://www.una.ac.cr/educare

educare@una.ac.cr

interdisciplinar, la educación genera una amplia ontología en los objetos de estudio (Cabrera, 2014; de Alba, 2003; Hoyos Medina, 1997; Moreno Bayardo, 2010; Naval, 2008; Sánchez Puentes, 1995; Weiss y Gutiérrez, 2003).

La forma en la cual se recrean las creencias particulares de las comunidades académicas puede darse desde posiciones teóricas disímiles. Tras la trama reticular de carácter simmeliano que planteaba la existencia diferenciada de la socialización (como forma de relación entre individuos, en la cual ponen en juego sus intereses) y la sociabilidad (como una forma instintiva -no material- que crea la dinámica de interacción). La sociología estableció un vínculo que entendía las relaciones sociales como determinadas por la estructura y no dependiente de los individuos. Gurvitch (citado por Chapman Quevedo, 2015) incluyó, en el análisis, el grupo particular como un microcosmos de la sociabilidad.

Gurvitch (1977) traza una escala de características de los grupos que va de lo superficial (concreto) a lo abstracto. Al definir los Social Frameworks (SF), pone en circulación marcos de interpretación social de carácter simbólico que corresponden a circuitos de relación y de referencia con capacidad vinculante entre los sujetos y la sociedad, los cuales transitan como entidades de vinculación o patrones de relación a través de los grupos o como formas sociales universales. Por otra parte, establece una relación entre las unidades de los SF, los niveles de profundidad de estudio y los diferentes conocimientos. En los dos primeros se puede manifestar cualquiera de los tipos de conocimiento que va de lo perceptual hasta lo filosófico. Mediante una tipología clasifica grupos o comunidades especializadas con lo cual se puede entender la sedimentación de las fuerzas simbólicas existentes en los procesos formativos (Gurvitch, 1977).

La teoría ofrece un marco para identificar las formas de socialidad, la jerarquización de valores y patrones sociales dominantes en el grupo. Las conceptualizaciones y las nuevas hipótesis se justifican por los criterios científicos y sociales en los cuales se producen. Se construye un proceso de cognición compartida en el grupo, para producir el conocimiento y la recreación de códigos o regulaciones sociales que fundan la validez de los productos científicos (Gurvitch, 1977).

Los elementos centrales de los SF de una comunidad científica se hacen patentes al identificar la existencia de objetos de estudio, la presencia de un cuerpo de especialistas, la aceptación de ciertas teorías y formas de organización del conocimiento acumulado, el despliegue de metodologías propias de investigación que se institucionalizan en una estructura organizativa (por ejemplo, departamentos o facultades) y muestran sus particularidades a través de las prácticas de formación y de realización de la investigación, donde prevalece una gestión que sostiene la convergencia e identidad del grupo (Becher, 1989; Krishnan, 2009).

El proceso central de los grupos académicos es la producción de la teoría, vincula los objetivos para describir, explicar e interpretar fenómenos específicos con la jerarquización de 
http://doi.org/10.15359/ree.25-3.13

http://www.una.ac.cr/educare educare@una.ac.cr

las problemáticas en su contexto. Al cultivarse entre las nuevas generaciones de investigación, se crea un puente entre lo macrosocial y la socialidad. Los SF se manifiestan en la aplicación del conocimiento a través de criterios, códigos y normas (Echeverría, 1995; Gurvitch,1977).

De esta manera, las formas de organización de los grupos académicos revelan una estrecha relación entre las tareas intelectuales, las formas de relación social en el interior del grupo y las necesidades del entorno. Se trata de una clausura basada en la identidad y en la determinación de fronteras simbólicas para facultar la exclusión de los miembros ajenos al grupo (Becher,1989; Gurvitch,1977).

Las propuestas de Becher (1989) y Gurvitch (1977) prevén la existencia de tres procesos centrales al interior de los grupos de formación: 1) un campo semántico amplio que abarca diferentes concepciones de teoría, de su clasificación como buena teoría y de su uso; 2) acciones de socialización que se manifiestan mediante normas, códigos, símbolos; 3) su apertura con el contexto.

Los campos disciplinares de los grupos de investigación son objetos de investigación primordiales. Pueden ser estudiados en sus procesos internos de producción científica o en el vínculo creado por los problemas del contexto, el progreso de las ciencias y los cambios en el significado de las teorías. Dicho de otra forma, las investigaciones podrían realizarse en torno a las prácticas del grupo y los procesos sociales con los cuales se establece la recuperación disciplinar del grupo de investigación y la dinámica histórica de prioridades institucionalizadas.

En este artículo, se aborda la educación a distancia como un subcampo disciplinar de la educación en el que se realiza investigación y docencia en torno a un objeto de carácter polisémico y en debate (Bosco Hernández y Barrón Soto, 2008), asumiéndola como un proceso educativo organizado, en constante cambio, y constituido como un sistema extendido en todos los niveles educativos, mediado por canales de comunicación e interacción que no poseen los límites de la presencialidad y sincronía y, que actualmente hace uso de las tecnologías digitales en forma intensiva.

\section{Metodología}

La población objeto de la investigación fue de estudiantes del Doctorado en Educación y del Doctorado en Sistemas y Ambientes Educativos de la Universidad de Guadalajara en México. Su elección se hace por su especialización en la formación de personal investigador en educación, tener amplio reconocimiento nacional y constituirse como un semillero para la investigación nacional. Un número elevado de su estudiantado egresado toma la educación virtual como el objeto de sus investigaciones.

Se analizaron los productos de ocho estudiantes (cuatro de cada doctorado) que muestran el desarrollo de los objetos de estudios a lo largo del primer año de formación. Su selección se hizo por la recomendación de los sujetos coordinadores de posgrado al asumirlo como 
http://doi.org/10.15359/ree.25-3.13

http://www.una.ac.cr/educare

educare@una.ac.cr

estudiantado destacado de su generación. Los textos y temas abordados con este grupo de estudiantes se centran en las acciones para delimitar el objeto de estudio; sus referentes son el anteproyecto de la investigación que se entrega al iniciar el proceso de formación, el avance del proyecto al concluir el primer semestre y el proyecto consolidado que se entrega al finalizar el segundo semestre. Pertenecen a la última generación de cada posgrado y estaban en la fase final de redacción de tesis.

La metodología se constituyó con dos estrategias básicas para la recolección y análisis de la información. En primerlugar, los productos estudiantiles se organizaron yanalizaron considerando la delimitación conceptual del tema abordado, la presencia y explicación de conceptos clave, las categorías de exploración del recorte en la realidad, la definición espaciotemporal de los sujetos de la investigación, las unidades de análisis y el procedimiento metodológico congruente con su marco teórico. Se utilizó un código binario (sí o no) para establecer si en el documento revisado se mostraba la presencia de cada rubro; si cumplía con los seis aspectos, conforme a los requerimientos de avance dispuestos en el perfil del programa curricular en cada posgrado: se podría afirmar que el objeto de estudio estaba consolidado. La tarea se hizo por el autor de este texto y se remitió a otro investigador para realizar una revisión semejante que funcionase como una opción de triangulación. Además, se hicieron entrevistas con el estudiantado que permitieron recuperar su experiencia en el período mencionado, para constituir una narración de la dificultad que le acarreó conformar el objeto de investigación. La entrevista se analizó, primordialmente, con la semántica de los constitutivos de la etapa del proceso de estudio y las dificultades percibidas por cada estudiante. El análisis consideró seis categorías relacionadas con el marco teórico aquí plasmado y su convergencia en el objeto de estudio: marco teórico, metodología, realidad, contexto, cultura académica.

En una segunda fase, se revisaron los programas de materias de ambos posgrados del primer año de formación con el propósito de identificar los contenidos, la lógica de estos y corroborar si existen acciones planteadas para clarificar la interdisciplinariedad, la revisión histórica de los objetos de estudio y la especialización disciplinar concurrente para enriquecer los objetos de estudio. Los programas fueron analizados como mediadores con el contexto.

La tercera tarea consistió en una revisión historiografía para reconocer la delimitación de fronteras disciplinares y los eventos históricos que contribuyeron a formar los campos de estudio especializados. En específico, se revisaron fuentes primarias y secundarias de acontecimientos y políticas educativas especializadas de la educación virtual, el conjunto de eventos del área que se convirtieron en problema según los diagnósticos oficiales $y$, finalmente, para el campo disciplinar se apeló a la historia de las disciplinas concurrentes en educación. La selección de textos fue abierta; dada la escasa producción temática, se buscaron a las autorías y textos más citados, privilegiando las investigaciones y ensayos académicos con estricta pertenencia al campo disciplinar. 
http://doi.org/10.15359/ree.25-3.13

http://www.una.ac.cr/educare educare@una.ac.cr

\section{Resultados}

Los datos iniciales se generaron mediante el estudio de textos a lo largo de un año. Se revisó el anteproyecto de ingreso al posgrado, el proyecto de investigación al concluir el primero y el segundo semestre de la formación. Al explorar la tercera versión del proyecto se pudo constatar que ninguno de los ocho proyectos cumplía con los requerimientos deseables de una definición del objeto de estudio. Por ejemplo, una de las alumnas (Bety) planteaba entre sus objetivos: Investigar y analizar los fenómenos en el proceso de enseñanza-aprendizaje en la formación de los estudiantes con relación al [sic] rendimiento académico en el curso de programación de la carrera de ingeniería en computación e informática del departamento (DV1.MT.P16).

Sin embargo, no definía el rendimiento académico en la teoría y no ubicaba en la metodología una estrategia para encontrar las causas del bajo rendimiento del alumnado. En cambio, sí proponía como un segundo objetivo: Diseñar un modelo B-learning como apoyo y estrategia de enseñanza utilizando el aula virtual (DV1.MT.P19).

Situaciones semejantes fueron comunes para todos los casos de estudio. En ellos, se evidenció total desconocimiento de la historia del problema y del tema, nula relación con el sistema educativo y poca profundidad teórica para atender el carácter interdisciplinar del objeto de la investigación. Si bien es cierto, a lo largo del tiempo, los proyectos presentaron una mejora, el objeto de estudio no pudo considerarse como un producto definido en esa etapa. El propio estudiantado admitió no tenerlo concluido en su primer año de formación.

En forma paralela, se hizo una revisión de los programas de materias del primer año para identificar aquellos que de manera explícita estableciesen actividades para favorecer la construcción interdisciplinar (ya fuese por medio de la teoría o la metodología) y, por otra parte, si existía un acercamiento bibliográfico a los acontecimientos históricos y a la evolución de los objetos de estudio inscritos en las áreas de especialización. En ambos posgrados, se evidenció que no había mediaciones para ello; en cambio, se identificó una dispersión teórica en los programas y la centralización de contenidos de metodología para la formulación de la investigación.

Considerando que el objeto de exposición en este artículo es la teorización sobre una articulación que vincule los procesos micro de formación con los elementos estructurantes del contexto, se deslizó el cuestionamiento de cuáles serían los principales acontecimientos que conforman el campo subdisciplinar de las políticas en la educación virtual. La búsqueda de información se perfiló para identificar las particularidades del campo especializado a través del desarrollo de las disciplinas, de los eventos históricos institucionalizados y de la presencia de políticas educativas pertinentes considerando antecedentes de investigación afines (Pacheco-Méndez, 2014). Aun cuando se asume la existencia de una relación entre las condiciones materiales del fenómeno que se estudia (por ejemplo, estrategias de enseñanza en la educación virtual durante la pandemia de Covid) y la articulación histórica del campo de 
http://doi.org/10.15359/ree.25-3.13

http://www.una.ac.cr/educare

educare@una.ac.cr

especialización disciplinar (por ejemplo, el estudio de las políticas de la educación virtual) es necesario profundizar en la mediación que tienen los temas de investigación en los programas emergentes, los enfoques teóricos para el abordaje de la interacción docente-estudiante y el aprendizaje. Así como el ajuste en la cultura académica para abordar estos factores. En este sentido, hay un propósito inserto en el texto para mejorar la comprensión de los factores estructurantes de los procesos formativos desde la teoría, contribuyendo con las investigaciones realizadas que demuestran la relación entre tradiciones y universos de conocimiento en las prácticas de formación, señaladas por Pacheco-Méndez (2014).

En la exploración de las condiciones de análisis para vincular los elementos macro, derivados de las ideas centrales que rigen la disciplina y las demandas históricas a la educación en el país, con los procesos micro que se asumen con la delimitación de fronteras de los grupos de formación es pertinente reflexionar sobre la omisión en el trabajo de Pacheco-Méndez (2014). Este no considera, en su investigación, la influencia que los problemas históricos del campo disciplinar y la reconfiguración teórica ejercen en los grupos de formación. En este sentido, es pertinente avanzar para identificar la diversidad y extensión de la información histórica que pudiera contribuir a la construcción de objetos de estudio.

En el caso educativo, las teorías son el componente central por el cual damos sentido a los acontecimientos específicos del proceso de enseñanza-aprendizaje y a los múltiples factores que los rodean. Sin embargo, los fenómenos educativos son multidisciplinares y cada teoría deriva de una ciencia diferente. Acercarse al desarrollo de sus conceptos, metodologías y enfoques permite comprender la relación disciplinar de la educación y la construcción de los objetos de estudio.

Al hacer un recuento histórico general es importante destacar la emergencia y consolidación del Estado para edificar la educación tal y como la conocemos. Este hecho, posibilitó el desarrollo de las disciplinas, propició el desarrollo de la investigación y a partir del siglo XX, facultó la reflexión para vincular diversos femémonos sociales y económicos con la esta. Según Archer (1979), el eje para la modernización del Estado deriva de las demandas de la Revolución Francesa (s. XVIII) y la Revolución Industrial (s. XIX) para armonizar la convivencia civil, desarrollar el crecimiento de las economías y aminorar la falta de conocimientos de las masas. La reflexión de estas necesidades impulsó las ideas centrales de la sociología educativa.

El núcleo duro de la discusión sociológica en la educación se conformó gracias al pensamiento de Marx, Weber y Durkheim, quienes propiciaron el debate sobre el carácter científico de la pedagogía, la importancia de la educación para la transformación social y la preservación de la cultura, en la revisión del papel del Estado para definir lo que se debe enseñar en las escuelas, ampliar la función de la educación para reducir las desigualdades en la sociedad y en su contribución a la economía. A través de sus propuestas, se cuestionó la burocratización de los procesos pedagógicos, la penetración del racionalismo para atender las necesidades de 
http://doi.org/10.15359/ree.25-3.13

productividad y el uso de la educación para mantener las formas materiales de vida y el sistema de ideas de una clase social particular. La consolidación de este núcleo de ideas permitió la formación de comunidades especializadas en temas como el carácter científico de la educación, la relación entre lo social y lo pedagógico, la educación para el trabajo versus la educación para la formación cultural, el rol de las burocracias en la educación, la relación educación-trabajo, el papel del Estado en la educación (Ramírez Díaz, 2020b).

Posteriormente, este núcleo conceptual se amplió gracias a las corrientes funcionalistas que ubicaron la educación como un subsistema necesario para mantener el orden en la sociedad. Se inicio el estudio de la inversión en la educación para propiciar el incremento de la productividad e inducir el desarrollo industrial. Con el arraigo de las metodologías cualitativas se incentivaron los estudios fenomenológicos y etno-metodológicos en la educación. En 1965, se llevó a cabo una investigación que consolidó la educación como ciencia. Gracias a los resultados presentados en el Informe Coleman, se hicieron notorios los múltiples factores que rodean los resultados en la educación, se redefinió la igualdad de oportunidades escolares y cobró importancia el estudio del conjunto de relaciones sociales entre individuos e instituciones formales para el ascenso en la pirámide social y la estratificación educativa (Fernández Enguita, 2016).

Con el desarrollo de la postura marxista se fundó un núcleo de estudio sobre la relación entre la educación y la construcción, reproducción y mantenimiento de las desigualdades sociales; la escuela como dispositivo disciplinario y la moral ortopédica; los trabajos centrados en la relación entre la educación, la socialización y la reproducción cultural. Se generaron numerosas críticas a la educación tradicional y a su lenta respuesta ante los incesantes cambios presentes en la sociedad. Ejemplo de ello es el colonialismo académico, los procesos de globalización (Ramírez Díaz, 2020b).

Otras disciplinas contribuyentes a la formación de la teoría de la educación son la pedagogía, la didáctica y la psicología educativa, muy cercanas entre sí por el interés de explicar el proceso de enseñanza-aprendizaje. Y tendrían un momento de convergencia con la sociología en los albores del siglo XX. En sus inicios, la pedagogía fue vista como una ciencia práctica en la búsqueda por encontrar la mejor forma de educar. Las teorías pedagógicas primarias surgen a partir de la reflexión sobre los fines de la educación. Su origen se ubica en las deliberaciones de Sócrates, Platón y Aristóteles en la Grecia antigua y las de Varrón, Cicerón, Horacio, Virgilio y Ovidio en Roma (Abbagnano y Visalberghi, 1964; Dilthey,1968).

La educación, hasta el Renacimiento, transitó entre el aprendizaje adquirido mediante lecturas de autores clásicos griegos y romanos y la transformación de la conciencia mediante el acercamiento a Dios, con un uso intensivo de métodos de enseñanza asentados en el dogma, la obediencia y el castigo. En el Renacimiento, la comprensión del mundo se modifica. Se creó una nueva concepción del ser humano y se dio paso a una separación entre las artes y la ciencia. En el 
http://doi.org/10.15359/ree.25-3.13

http://www.una.ac.cr/educare

educare@una.ac.cr

siglo XVI, gracias a la Reforma protestante, se separa al Estado de la Iglesia, con ello, la voluntad y lo humano como parte de la educación se instala fuera de la esfera de Dios (Abbagnano y Visalberghi, 1964; Dilthey, 1968).

El XVII es, para Dilthey (1968), el siglo de la consolidación de la pedagogía y la didáctica que busca hacer frente a problemas como la pobreza y el incremento de niñez sin educación. Comenio impulsa la idea de fundar escuelas que incluyan a la totalidad de miembros de la sociedad. Se genera un fuerte debate sobre si el origen de las ideas y el conocimiento es innato, para dar paso al estudio del aprendizaje y la cognición.

En el siglo XVIII, con la divulgación de las ideas de la llustración, se allanó el camino al desarrollo de la ciencia, al satisfacer la demanda masiva de educación y buscar nuevas formas de gobierno alternativos a la monarquía. En este el período la pedagogía recibe un impulso con el naturalismo de Rousseau y con los escritos de Kant al enfatizar el respeto a la ley moral y la posibilidad de regirse por las constricciones morales, la razón práctica y la libre investigación científica (Abbagnano y Visalberghi, 1964).

El siglo XIX es considerado el siglo del nacionalismo, el industrialismo y el capitalismo. Las ideas pedagógicas y sociales en torno a la educación empiezan a vincularse. Humboldt reflexiona sobre las funciones del Estado e impulsa la idea del desarrollo educativo individual libre de la influencia estatal. En Francia se crean escuelas para la educación popular, la instrucción técnico-profesional y superior. Pestallozi muestra inquietud por impulsar la autonomía moral en la educación y neutralizar los métodos impositivos y restrictivos dirigidos a cultivar el intelecto. Fröebel se preocupa por el desarrollo psíquico del estudiantado mediante el uso de los juegos. Herbart desarrolla una visión psico-educativa que asocia representaciones básicas con otras para formar tendencias representacionales y actos de apetito, voluntad o cognoscitivas (Abbagnano y Visalberghi, 1964).

Entre los siglos XIX y XX, se propicia la fusión del núcleo duro de las teorías de la educación. Los contenidos fundados en la sociología se empiezan a imbricar con los de la pedagogía y la psicología. En los albores del siglo XX, se difunden trabajos que relacionan la psicología y enseñanza, lo cual, resulta clave para formar un campo interdisciplinar sobre el aprendizaje. Se desarrolla y operacionaliza la teoría sobre la inteligencia, las capacidades y el avance en la escuela (Ramírez Díaz, 2020b).

El siglo XX es el escenario del desarrollo de pedagogías con la tutela del Estado. Se promueve la enseñanza de las ciencias basadas en la idea de evolución y en el positivismo. Surgen las pedagogías pragmáticas que subordinan el conocimiento a la acción. La especialización de la psicología en la educación incorporo las aportaciones del conductismo, el paradigma sociocultural y la epistemología genética para diferenciar los problemas educativos emergentes con las demandas planteadas por el crecimiento económico y la masificación escolar (Ramírez Díaz, 2020b). 
http://doi.org/10.15359/ree.25-3.13

Para el último cuarto del siglo $\mathrm{XX}$, el desarrollo teórico interdisciplinar de la teoría de la educación ya estaba consolidado. El corpus teórico era un referente para el abordaje de problemas específicos en la época. Los campos disciplinares mostraban un soporte de procesos científicos para solventarlos, la presencia de un lenguaje especializado que originó la formación de una identidad a los sujetos productores de teoría y su público usuario.

El desarrollo de la pedagogía, junto con la psicología y la sociología, se consolidó en la medida en que el Estado se iba apropiando de las funciones de administrar la educación en sociedades con enormes poblaciones de niñez y juventud en condiciones de vida precarias. También, por la responsabilidad asumida para promover la investigación sobre los problemas de la enseñanza en los sistemas educativos masivos y con demandas latentes del desarrollo económico. Las implicaciones que esto tenía en la formación de docentes, y en la atención de las diferencias individuales y de los grupos según su clase social, nivel cultural o desarrollo psicológico y moral dentro de las condiciones políticas, sociales y económicas de cada país. En su conjunto, la apropiación estatal de la organización de la educación, el desarrollo teórico interdisciplinar y las condiciones materiales constituyeron un catalizador de problemas para ser abordados por la investigación educativa y dieron la pauta para conformar objetos especializados de la educación no presencial.

Consecuentemente, para franquear la formación de un campo disciplinar especializado como lo es la educación a distancia, es necesario pasar del marco histórico general de la teoría de la educación al especifico de una subdisciplina. La construcción de este escenario transita por una narración histórica de los acontecimientos educativos que les dieron forma a sus fronteras, y este es un proceso particular en cada país. Si bien es cierto, las teorías de la educación en sentido abstracto son universales, la manera en cómo son recibidas y apropiadas en los grupos subdisciplinares depende de dos aspectos centrales: las dinámicas internas de las comunidades académicas y los problemas o necesidades de los sistemas educativos nacionales.

En el caso particular que se revisa, los elementos considerados indispensables para relevar la educación a distancia son: la responsabilidad del Estado asumida para abatir el analfabetismo y contar con el número suficiente de maestras y maestros capacitados en la educación básica; el impacto del uso de las TIC que derivó en entidades de apoyo a la educación; su inclusión en las políticas de Estado para incrementar la matrícula o atender problemas sociales en la educación superior. Si bien es cierto, estos temas se encuentran ausentes de los programas de estudio de los posgrados revisados, es necesario debatir sobre el impacto que puede tener el ejercicio individual para puntualizar su relación en el objeto de estudio que construye. A continuación, se hace una cronología genérica para el caso de México.

La legitimación social de la función educativa del Estado postrevolucionario en México se consolidó con: a) el reconocimiento como autoridad para promulgar las políticas y leyes educativas; b) la conformación de un sistema masivo de educación con procesos especializados; 
http://doi.org/10.15359/ree.25-3.13

http://www.una.ac.cr/educare

educare@una.ac.cr

c) la delimitación de las prácticas escolares a través de dispositivos de organización educativa, normas y cultura especifica. Esto se construyó gracias a diversos acontecimientos en el tiempo.

A partir de 1917, los ideales de la educación mexicana (laica, gratuita y obligatoria) fueron plenamente incorporados al ideario constitucional. Con la creación de la Secretaría de Educación Pública (SEP), en julio de 1921, se reafirmó la potestad estatal en materia educativa, el asentamiento de las bases para conformar un aparato burocrático especializado que gestionaría los recursos y la información del sector (Loyo y Staples, 2010; Solana et al., 2011).

En su momento, la SEP buscaba soluciones a los problemas derivados del analfabetismo, la multiculturalidad, el multilingüismo y la demanda de personal capacitado por el crecimiento de económico. Alternativamente gestionó investigación para solucionar los problemas nacionales derivados de los requerimientos que el proceso de reconstrucción y desarrollo económico implicaban (Solana et. al., 2011).

Su principal objetivo fue abatir el analfabetismo de la población mexicana, pero enfrentaba la carencia de maestros y maestras. El primer intento institucional para resolver estos problemas se dio al crear en 1923 el Plan de las Misiones Federales de Educación. Las misiones incluyeron los principios didácticos de Dewey. Intentaban formar escuelas para la vida y capacitar al personal docente en las propias comunidades, con el apoyo de docentes ambulantes. Pese a los esfuerzos realizados, no se alcanzaron los resultados esperados. Cuando llegó Lázaro Cárdenas a la Presidencia dispuso un cambio en la educación al modificar el Artículo tercero de la Constitución Mexicana (1934), y señalar que sería socialista y formaría la racionalidad en la juventud. A partir de 1935, se empezaron a utilizar principios de educación basados en la pedagogía socialista, se crearon escuelas regionales campesinas, se reorganizaron las normales para la formación de los nuevos maestros y maestras y se unificó la producción editorial dirigida a docentes mediante la revista El maestro rural que se complementó con cursos por correspondencia y la serie Simiente (Navarrete-Cazales y Manzanilla-Granados, 2017).

Las estrategias de gobierno para estas capacitaciones se formalizan en 1944, al ser creado el Instituto Federal de Capacitación del Magisterio. Para 1948 se formó el Servicio de Educación Audiovisual de la Dirección General de Enseñanza Normal y el Departamento de Enseñanza Audiovisual, instancias que producían material didáctico y colaboraban en la formación de docentes (Bosco Hernández y Barrón Soto, 2008).

Con la creación del Instituto Latinoamericano de la Cinematografía Educativa (ILCE) (1954-1956) se dio impulso a la producción de materiales didácticos con uso de la TV y el cine. En 1955, la UNAM y el IPN transmitían programas educativos y culturales; además se utilizó la radio de onda corta (XEUNT) para escuelas de la sierra Tarahumara. A partir de 1965, se intentó mejorar el nivel de académico del estudiantado e incrementar la alfabetización de la población con el uso de series de televisión, lo cual luego, en 1970 se convertiría en el proyecto educativo 
http://doi.org/10.15359/ree.25-3.13

de Telesecundaria. En 1971, se fundó la Televisión Rural de México (TRM) (Bosco Hernández y Barrón Soto, 2008).

En el nivel superior, en la medida que crecía la demanda, se buscó aumentar y mejorar la organización de la educación a distancia. La UNAM fortaleció su Sistema de Universidad Abierta (SUA) en 1972, se creó el Colegio de Bachilleres (1973) con un sistema de enseñanza abierta que prestaba asesoría telefónica o postal. La Universidad Pedagógica Nacional (1973) capacitó maestros y maestras con un sistema educativo a distancia y en 1974 el IPN organizó un sistema abierto de enseñanza para los niveles medio superior y superior. En 1978, el gobierno impulsó el Consejo Coordinador de Sistemas Abiertos (1978). En 1981 se funda El Instituto Nacional de Educación de Adultos (INEA) para atender los problemas de analfabetismo y rezago educativo. Con el arribo de las TIC y el uso generalizado del internet entre la población se daba pie a nuevas modalidades de educación a distancia (Bosco Hernández y Barrón Soto, 2008).

La educación a distancia se mencionó en las políticas educativas en el Plan Nacional de Educación (1978) con el uso de audiovisuales para la educación. En 1996, se instrumentó el Programa de Educación a Distancia (PROED) derivado del Programa de Desarrollo Educativo (1995-2000) y en el Programa Nacional de Educación (2001-2006) se propuso usar los sistemas modernos de información y comunicación para favorecer la equidad de la educación superior mediante la ampliación de la oferta de programas impartidos a distancia en las regiones de baja densidad poblacional. A partir 2007, los problemas diagnosticados en los instrumentos de política educativa focalizaban su atención en la caída de la cobertura y la baja calidad de los estudios en el nivel superior, así como en la desvinculación entre mercado laboral y universidades. Para solventarlos, se propuso intensificar la educación mediada por tecnologías para dispersarla en diferentes regiones del país que no contasen con universidades, pero se estableció la necesidad de monitorear la calidad de los programas mediante procesos de certificación con criterios mínimos para garantizar una buena enseñanza (Ramírez Díaz, 2011). Posteriormente, en los dos siguientes sexenios, se mantuvo una posición de inclusión de la educación a distancia como estrategia de equidad por medio del incremento de matrícula.

La trayectoria histórica revisada en el desarrollo de las diferentes disciplinas convergentes en educación y el eje de problemas asociados a la educación virtual que se ha mostrado son un punto de irrupción para discutir su posible influencia en los grupos de formación de personal investigador sobre la educación virtual, su peso en la conformación de los objetos de estudio, las formas en que se toma conciencia de su importancia para el desarrollo de la investigación y sus mecanismos de institucionalización en los grupos particulares de formación. Un punto de alta relevancia sería establecer las mediaciones para que el estudiantado realice una revisión histórica de las formas materiales del objeto de estudio, las concepciones teóricas de su abordaje y la dinámica estatal asumida por el gobierno para gestionar el problema. 
http://doi.org/10.15359/ree.25-3.13

http://www.una.ac.cr/educare

educare@una.ac.cr

Junto a los aspectos mencionados en el párrafo anterior habría que enfatizar la importancia de las culturas académicas en la formación de personal investigador, pues estas establecen las jerarquías de valor en los productos académicos y del uso pertinente de teorías y metodologías en las diferentes etapas de la investigación.

El trabajo de Pacheco-Méndez (2014) pudo demostrar afinidad entre las prácticas de formación en doctorados de España, Francia y México, las cuales se encuentran influenciadas por contextos de mediación donde se mezclan aspectos históricos de la formación de los grupos, lo social, lo institucional y lo científico. Sin embargo, como ya se dijo, no fueron asociadas a las problemáticas históricas específicas de cada sistema educativo ni a la apropiación interdisciplinar en los grupos de formación.

\section{Discusión}

Es difícil negar la dificultad que acarrea la consolidación de los objetos de investigación para el estudiantado de posgrado en educación. Los datos obtenidos en el estudio muestran que se desarrolla mediante un proceso individual apoyado por lecturas de metodología y fuertemente influenciados por la cultura y las prácticas de los grupos de formación; sin embargo, se omiten las estrategias de articulación disciplinar y los contenidos históricos concurrentes con los temas específicos de investigación del estudiantado.

Al realizar la investigación empírica en los procesos grupales internos y al emplear los SF como base teórica del trabajo, se hace admisible incorporar una diversidad de fuerzas simbólicas sobre los procesos formativos. En este caso, se pretende relevar las problemáticas empíricas históricas del sistema educativo y la interdisciplinariedad que se teje en la subdisciplina. Asumidas como factores inadvertidos en la formación del personal investigador, se podría hipotetizar para vincularlas a las dinámicas del proceso de enseñanza-aprendizaje y a su concatenación con la delimitación que hacen las culturas de las comunidades académicas. En este sentido, teóricamente, se crea una dimensión extra de estudio: el impacto del estudio de la historia de la interdisciplina educativa y de los eventos históricos del sistema educativo nacional (SEN) como un marco de referencia que pasa por encima de las tribus académicas para reconocer el valor de la historia en la producción del conocimiento científico.

Sin embargo, el detalle de los resultados obtenidos en estudiantes de ambos posgrados muestra un proceso de formación de carácter individual, comprometido con una estructura de actividades de formación enquistada, con ritmos intensos de lectura y nula asociación con la historia de los problemas y de los conceptos interdisciplinares que han servido para formular las explicaciones del fenómeno educativo.

Los resultados de la investigación mostrados no aspiran a dar una solución universal y práctica para cada contexto de formación de personal investigador de la educación. Pues, 
http://doi.org/10.15359/ree.25-3.13

en cada persona, existen mediaciones específicas que hacen único e irrepetible el proceso. Lo que se pretende relevar es la necesidad de fortalecer los marcos teóricos y metodológicos para investigar el proceso de enseñanza aprendizaje no presencial. En una recomendación panorámica basada en la constitución de los SF, se pueden considerar los siguientes aspectos: el tema de investigación del estudiantado y el espacio socio temporal en que lo ubica, su desarrollo histórico como problema educativo, la importancia que le concede el Estado a lo largo del tiempo, el background del estudiantado, la disciplina eje del estudio (pedagogía, didáctica, psicología educativa, sociología educativa o filosofía de la educación) y su relación con las otras disciplinas a través de los conceptos o las metodologías, las características del programa de formación (lo que incluye el diseño de contenidos de las materias y las formas valoradas de enseñanza), la cultura del grupo académico en el cual se inscribe el programa de formación y las características (capacidad y capital social -cultural) de los actores de la educación.

Lo señalado en el párrafo anterior ha sido estudiado y problematizado por la investigación de la formación de personal investigador y también es conocido, en términos generales, por la mayoría de las personas formadoras de personal de investigación. Sin embargo, lo que está prácticamente abandonado es el reconocimiento del peso de la evolución teórica disciplinar y los acontecimientos históricos institucionalizados en los SEN para contribuir a la formación de los objetos de estudio, lo que podría traducirse en su incorporación en el currículo o en la toma de conciencia del director o directora de tesis para complementar el diseño.

Si se asume una relación simbólica entre la evolución de las disciplinas y la historia de los acontecimientos particulares en los SEN, con las culturas y los contextos recreados en las tribus académicas, y de ambos con los procesos educativos en el aula, tendríamos una construcción de significados para una tarea concreta (como es la conformación del objeto de estudio) en tres dimensiones, tal y como lo propone Gurvitch (1977) en su teoría: lo macro (reflejado en los aspectos históricos), lo medio (presente en la cultura del grupo y la curricula dispuesta) y lo micro (en los significados filtrados en las prácticas de enseñanza-aprendizaje).

Entre los temas de discusión que de esta propuesta podrían revelarse, se considera importante conocer las características de las organizaciones que operan la educación a distancia e identificar el impacto de su dinámica social y cultural en los procesos de educación; identificar las corrientes pedagógicas, los cambios didácticos y su posicionamiento teórico de lo que es la educación en los programas de formación de docentes, el diseño curricular, los programas de materia y los diseños de cursos; el análisis de las políticas educativas y su precisión para diagnosticar los problemas y necesidades del campo disciplinar y la pertinencia de la instrumentación política para atender las necesidades de la educación a distancia.

El reto es empezara desarrollar trabajo de investigación empírico que someta a cuestionamiento los principios teóricos que aquí se plantean para su discusión. Solo a manera de ejemplo, se presentan 
http://doi.org/10.15359/ree.25-3.13

http://www.una.ac.cr/educare

educare@una.ac.cr

un par de temas que podrían abordarse para el caso específico de la educación a distancia que involucran un trabajo en las tres dimensiones señalados en párrafos anteriores:

El tema de un análisis del impacto o descripción de las políticas adoptadas para fortalecer la educación a distancia en el nivel superior podría convertirse en un objeto de estudio sólido a través de la caracterización y precisión histórica de los diagnósticos de las políticas educativas para definir los programas de fortalecimiento de la educación superior a distancia en México y su huella en las instituciones educativas. En términos disciplinares podría abordarse desde las políticas públicas (la revisión histórica de los métodos empleados en el ciclo de políticas) o las teorías organizacionales (los marcos conceptuales históricos que explican el cambio institucional).

En el caso de la capacitación de docentes con el uso de métodos de educación a distancia se podría fortalecer, como objeto de estudio, con el análisis de los métodos de capacitación que a lo largo del tiempo se han implementado para atender a los maestros y maestras de México. Una revisión histórica de las disciplinas involucradas podría analizar los principios pedagógicos implícitos en los materiales y los medios de capacitación empleados históricamente en México como, por ejemplo: radio, bibliotecas ambulantes, capacitación masiva hasta llegar a los actuales cursos que se imparten con uso intensivo de la tecnología entre los cuales se manejan: cursos en línea, Webinar, uso de redes sociales. Desde la psicología educativa se pueden analizar los principios de aprendizaje de cada curso de capacitación.

La inclusión de los aspectos referidos es una propuesta para dar forma a la idea del desarrollo del "pensamiento epistémico". Sin embargo, siempre habrá que considerar su dependencia con los aspectos culturales y estructurales de los contextos de formación y de sus agentes. Para este autor, es razonable problematizar el tema y desarrollar investigación que pueda arrojar información para contribuir a la mejora de los procesos de formación de personal investigador en educación.

\section{Conclusiones}

La propuesta teórica que aquí se presentó intentó trazar el desarrollo histórico de las disciplinas convergentes en educación y los eventos más representativos en la historia de la educación nacional como un elemento central de la creación de fronteras para explorar la creación de comunidades especializadas en la producción y la formación de personal investigador de la educación a distancia.

A partir de los datos obtenidos puede afirmarse que el campo disciplinar de la investigación para la educación, y en específico sobre la educación a distancia, construye sus fronteras gracias a un conjunto de eventos históricos y al desarrollo de diversas disciplinas. Se trata de un tejido vinculante de la teoría, eventos históricos de la educación en México y la emergencia de políticas 
http://doi.org/10.15359/ree.25-3.13

educativas. Sin embargo, se considera que es un ámbito con bajo volumen de investigación, en términos teóricos y empíricos, que ayude a comprender las aportaciones de la teoría de la educación a la solución de problemas educativos.

Al observar la reserva por realizar investigación centrada en los procesos y acciones que se llevan a cabo en la formación de personal investigador para identificar la relevancia de la discusión interdisciplinar y la historia de los eventos históricos en la educación, puede hipotetizarse que su ausencia representa una limitante para la construcción de los objetos de estudio.

La propuesta que se ha planteado tiende a ser más de carácter teórico que empírico. La principal recomendación sería el continuar con más investigaciones para corroborar o refutar los hallazgos encontrados. Al producir más teoría sobre el tema se estaría en condiciones de realizar modificaciones curriculares puntales y fortalecer en un sentido especifico a los equipos interdisciplinares para formular acciones dialógicas de formación.

Con el texto escrito se presta atención a un conjunto de problemas alternativos a los señalados por especialistas en la formación de personal investigador y se considera fundamental reconocer el entramado interdisciplinar de la sociología, la psicología, la pedagogía y la didáctica como generadores de temas y problemas de educación. Asimismo, se considera trascendente registrar la relación de los objetos de investigación con los eventos históricos de la educación nacional: la función del Estado en la organización y desarrollo de la educación a distancia, la capacitación de los maestros y maestras para los procesos de enseñanza-aprendizaje y los cambios generados en la educación por el uso de la tecnología.

Desde un posicionamiento teórico, se considera que los Social Frameworks son un concepto útil para profundizar en el proceso de desarrollo de los grupos de formación de personal investigador en educación a distancia e identificar sus características en la dinámica vinculatoria de las necesidades sociales y económicas con la cultura y normatividad propias de los grupos de formación que orientan la formación. Para ello, se requieren estudios empíricos sobre las formas en que se recrean los procesos de formación a través de lo curricular, los programas de estudio y los programas de materia y cómo se ven influenciados por las organizaciones y políticas especializadas.

El incremento de la cantidad y la calidad de la producción sobre la educación a distancia depende tanto de los esfuerzos de los grupos investigadores consolidados como de la mejora de los procesos de formación de personal investigador. En ello, es importante reconocer la característica particular de la educación a distancia por el uso intensivo de las tecnologías de la información en la sociedad y su influencia en la educación; todo ello propiciado por el desarrollo acelerado de la tecnología que confiere una nueva representación conceptual a la sociedad. 
http://doi.org/10.15359/ree.25-3.13

http://www.una.ac.cr/educare

educare@una.ac.cr

\section{Declaración de Material complementario}

Este artículo tiene disponible, como material complementario:

-La versión preprint del artículo en https://doi.org/10.5281/zenodo.4042032

\section{Referencias}

Abbagnano, N. y Visalberghi, A. (1964). Historia de la pedagogía. FCE.

Archer, M. S. (1979). Social origins of educational systems. Sage Publications.

Becher, T. (1989). Tribus y territorios académicos. La indagación intelectual y las culturas de las disciplinas. Gedisa.

Bosco Hernández, M. D. y Barrón Soto, H. S. (2008). La educación a distancia en México: Narrativa de una historia silenciosa. UNAM. http://ru.ffyl.unam.mx/bitstream/10391/3714/1/Bosco Barron Educacion a distancia Mex 2008.pdf

Cabero Almenara, J. (2008). La investigación en la educación a distancia en los nuevos entornos de comunicación telemáticos. Revista Internacional de Ciencias Sociales y Humanidades. SOCIOTAM, 18(2), 13-34. http://www.redalyc.org/pdf/654/65411193002.pdf.

Cabrera, D. M. (2014). La institucionalización de la investigación educativa en México: Cuatro ángulos de abordaje. Revista del/ICE, (35), 19-33. http://revistascientificas.filo.uba.ar/index. php/iice/article/view/1958

Castellanos, J. M. (2016). Reflexión acerca de la enseñanza de la investigación en tres programas de formación: Antropología, Sociología y Maestría en Ciencias Sociales (MACISO). En C. Gallegos Elías, A. Mejía Martínez y Y. Paredes Vilchiz (Coords.), ¿Cómo investigamos? ¿Cómo enseñamos a investigar? (Tomo 4, pp. 95-128). UNAM. https://www.unamenlinea.unam. $\mathrm{mx} /$ recurso/83997-como-investigamos-como-ensenamos-a-investigar-in-memoriam-ahugo-zemelman

Chapman Quevedo, W. A. (2015). El concepto de sociabilidad como referente del análisis histórico. Investigación \& Desarrollo, 23(1), 1-37. https://www.google.com/url?sa=t\&rct=j $\& q=\& e s r c=s \&$ source $=$ web\&cd $=1 \& v e d=2$ ahUKEwjowsP $8 Y$ IAhUMnaOKHTEcCOUQFjAAe gQIARAC\&url=http\%3A\%2F\%2Fwww.redalyc.org\%2Fpdf\%2F268\%2F26839041001.pdf\& usg=AOvVaw2rarTvDtkrRvhnK2M9NFj4

De Alba, A. (Coord.). (2003). Filosofía, teoría y campo de la educación. Perspectivas nacionales y regionales. COMIE-CESU-SEP. 
Dilthey, G. (1968). Historia de la pedagogía. Editorial Losada.

Echeverría, J. (1995). Filosofía de la ciencia. AKAL.

Fernández Enguita, M. (2016). El informe Coleman: Una lección de sociología y de política. Revista de Sociología de la Educación, 9(1), 37-45. https://ojs.uv.es/index.php/RASE/article/ view/8402

Gurvitch, G. (1977). The social frameworks of knowledge. Harper \& Row.

Hoyos Medina, C. Á. (Coord.). (1997). Epistemología y objeto pedagógico. ¿Es la pedagogía una ciencia? CESU. Plaza y Valdez Editores.

Krishnan, A. (2009). ¿What are academic disciplines? Some observations on the disciplinarity vs. interdisciplinarity debate. NCRM. Working Paper. http://eprints.ncrm.ac.uk/783/1/what are academic disciplines.pdf

Loyo, E. y Staples, A. (2010). Fin del siglo y de un régimen. En D. Tanck (Coord.), Historia mínima. La educación en México (pp. 127-153). El Colegio de México.

Martín Rodríguez, E. (1993). La investigación sobre educación a distancia en el ámbito Iberoamericano: Sus características, avances y retos. Revista Iberoamericana de Educación a Distancia, 6(1), 7-26. http://revistas.uned.es/index.php/ried/article/view/21060/17740

Moreno Bayardo, M. G. (2010). Historias de formación para la investigación en doctorados en educación. Plaza y Valdez.

Naval, C. (2008). Teoría de la educación. Un análisis epistemológico. EUNSA.

Navarrete-Cazales, Z. y Manzanilla-Granados, H. M. (2017). Panorama de la educación a distancia en México. Revista Latinoamericana de Estudios Educativos, 13(1), 65-82. https://www. redalyc.org/pdf/1341/134152136004.pdf

Pacheco-Méndez, T. (2014), Tradición, contexto y objeto de estudio en las tesis doctorales en educación de tres universidades. Revista Iberoamericana de Educación Superior, 5(12), 4669. https://doi.org/10.22201/iisue.20072872e.2014.12.109

Ramírez Díaz, J. A. (2011). Políticas y escenarios de futuro para la educación virtual en la educación auperior de México. Apertura, especial, 104-117. http://www.udgvirtual.udg.mx/apertura/ index.php/apertura/article/view/228/243

Ramírez Díaz, J. A. (2020a). El CONACYT como organismo rector de las capacidades de Estado en la relación de la ciencia con el sector educativo (Proyecto y síntesis de resultados). Universidad de Guadalajara. Inédito. 
http://doi.org/10.15359/ree.25-3.13

http://www.una.ac.cr/educare

educare@una.ac.cr

Ramírez Díaz, J. A. (2020b). Configuraciones disciplinares para la producción científica en la educación de México. Una revisión desde la sociología de la educación y la formación del sistema educativo. Nóesis, 30(58), 1-31. https://doi.org/10.20983/noesis.2020.2.1

Sánchez Puentes, R. (1995). Enseñar a investigar. Una didáctica nueva en la investigación de las ciencias sociales y humanas. UNAM.

Siemens, G., Gašević, D. y Dawson, S. (2015). Preparing for the digital university: A review of the history and current state of distance, blended and online learning. doi.org/10.13140/ rg.2.1.3515.8483

Solana, F., Cardiel Reyes, R. y Bolaños, R. (Coords.). (2011). Historia de la educación pública en México (1876-1976). FCE.

Weiss, E. y Gutiérrez, N. G. (2003). Comunicación de la investigación educativa (Capíutlo 7). En E. Weiss (coord.), El campo de la investigación educativa 1993-2001 (Vol. 1, pp.169-192). COMIE. https://www.academia.edu/7299435/El campo de la investigaci\%C3\%B3n educativa?auto=download

Zemelman, H. (2004). Pensar teórico y pensar epistémico: Los retos de las ciencias sociales latinoamericanas. IPEGAL https://repository.unad.edu.co/bitstream/10596/5564/1/ Documento7.pdf

Zemelman, H. (2011). Conocimiento y sujetos sociales. Contribución al estudio del presente. Instituto Internacional de Integración del Convenio Andrés Bello (III-CAB). https://www. academia.edu/30356942/Conocimiento y sujetos sociales contribuci\%C3\%B3n al estudio_del_presente_Zemelmam_Hugo 